Arq. Bras. Med. Vet. Zootec., v.69, n.1, p.252-258, 2017

\title{
Utilização da técnica in vitro semiautomática de produção de gases na avaliação da fermentação microbiana do ceco de suínos
}

\author{
[Use of the semi-automatic in vitro gas production technique for the evaluation of swine cecal \\ microbial fermentation] \\ C.A. Silva Júnior ${ }^{1}$, S.L.S. Cabral Filho ${ }^{2}$, F.L. Silva ${ }^{3}$, K.M. Silva ${ }^{4}$, A.R.R. Cabral ${ }^{4}$, T.F. Braga ${ }^{1}$, \\ F.N.G. Costa ${ }^{4}$, R.D. Navarro ${ }^{2}$, L.S. Murata ${ }^{2}$ \\ ${ }^{1}$ Aluno de pós-graduação - Universidade de Brasília - UnB - Brasília, DF \\ ${ }^{2}$ Faculdade de Agronomia e Medicina Veterinária - UnB - Brasília, DF \\ ${ }^{3}$ União Pioneira de Integração Social - UPIS - Brasília, DF \\ ${ }^{4}$ Bolsista - Universidade de Brasília - UnB - Brasília, DF
}

\begin{abstract}
RESUMO
O presente estudo teve como objetivo avaliar o os padrões de fermentação microbiana do ceco de suínos, utilizando-se um modelo aplicado na técnica in vitro de produção de gases para ruminantes. Três tipos de inóculo foram utilizados: ceco de suínos criados ao ar livre (CSF), ceco de suínos criados confinados (CSC) e líquido ruminal de bovino (LRB). Os substratos utilizados derivaram de uma dieta de suínos à base de farelo de soja e de grãos de milho, atendendo as exigências nutricionais dos suínos. Para composição dos substratos, foram estabelecidos diferentes níveis de substituição da dieta basal pela torta da amêndoa da Acrocomia aculeata (AA), coproduto da produção do biodiesel, sendo: BAS - 100\% de dieta basal; M10 - 90\% de dieta basal e $10 \%$ AA; M20 - 80\% de dieta basal e 20\% AA e o TF - feno de Tifton 85 (Cynodon spp.). A produção de gases foi avaliada nos tempos zero, três, seis, nove, 12, 16, 24, 48, 72 e 96 horas após a incubação. Foram analisados o potencial máximo de produção de gás (A) e o tempo de colonização $(\mathrm{L})$. O delineamento experimental utilizado foi inteiramente ao acaso, em arranjo fatorial 4 x 3 . Os dados de A e $\mathrm{L}$ foram submetidos à análise de variância, e as médias comparadas pelo teste de Tukey a 5\%. Comparando os inóculos para A (mL/gMS), LRB e CSF apresentaram valores similares, diferindo apenas para TF. O tempo de colonização (L) entre inóculos, o CSC e o CSF apresentaram os menores tempos de colonização quando a ração foi BAS. Com os substratos M10 e M20, o inóculo LRB apresentou o menor tempo, CSF o maior tempo e CSC não diferiu de ambos. Não houve diferença significativa entre os inóculos para o substrato TF. A técnica de produção de gases utilizada para o ceco de suínos apresentou resultados semelhantes aos encontrados para ruminantes. O modelo matemático usado foi adequado para descrever a curva de fermentação no ceco de suínos, mostrando semelhanças entre as microbiotas do ceco e do rúmen.
\end{abstract}

Palavras-chave: alimentos alternativos, fibra dietética, potencial fermentativo

\begin{abstract}
This study aimed to evaluate the microbial fermentation patterns of the pig ceca using the technique of in vitro gas production for ruminants. Three types of inoculums were used: swine ceca raised in a free range system $(C S F)$, swine ceca raised in a conventional confined system (CSC) and bovine rumen fluid (LRB). The substrates used were derived from pig's diet with soybean meal and corn grits, according to the nutritional requirements of swine. The substrates were composed by different set levels of substitution of basal diet by meal almond Acrocomia aculeata (AA), as: BAS - 100\% of basal diet; 110 - 90\% of basal diet and 10\% AA (M10); M20 - 80\% of basal diet and 20\% AA (M20) and TF - Tifton 85 hay (Cynodon spp.).The gas production was evaluated at times 0, 3, 6, 9, 12, 16, 24, 48, 72 and 96 hours after incubation. The study analyzed the maximum potential of gas production $(A)$ and lag time $(L)$. The experimental design was completely randomized in a $4 \times 3$ factorial arrangement. The $A$ and L data were submitted to analysis of variance and the averages compared by Tukey 5\% test. Comparing the inoculum for A ( $\mathrm{ml} / \mathrm{gDM}), \mathrm{LRB}$, and CS, they showed similar values, differing only for TF. The lag time (L) between inoculum, the CSC and CSF had the lowest
\end{abstract}

Recebido em 31 de julho de 2015

Aceito em 18 de fevereiro de 2016

E-mail: juniorc10@hotmail.com 
Utilização da técnica...

colonization times when the meal was BAS. With M10 and M20 substrates, the LRB inoculum showed the lowest time, CSF had the longest time and CSC did not differ from both. There was no significant difference between the inoculum for TF substrate. The gas production technique used for the ceca of pigs showed similar results to those for ruminants. The mathematical model used was adequate to describe the fermentation curve in the ceca of pigs, showing similarities between the microbiota of the ceca and the rumen.

Keywords: alternative feeds, dietary fiber, fermentation potential

\section{INTRODUÇÃO}

O interesse de se utilizarem ingredientes fibrosos como fonte de energia na suinocultura não é um conceito novo, pois vem sendo discutido por pesquisadores desde os anos 30. Porém, a pesquisa sobre o potencial da fibra dietética usada na produção de suínos necessita de uma quantificação e identificação por meio da medição das interações entre os efeitos fisiológicos associados com a digestibilidade, causados pela variável conteúdo fibroso que exista entre as diversas matérias-primas (Gomes et al., 2007).

Segundo Brouns et al. (1994) e Ramonet et al. (1999), apesar de os suínos serem animais não ruminantes e não possuírem a mesma capacidade de digerir a fração fibrosa do alimento com a mesma eficiência de um animal ruminante, a fibra dietética vem sendo utilizada como fonte de energia para os monogástricos, em especial para suínos destinados ao abate, machos reprodutores, marrãs e fêmeas em gestação.

O uso da fibra dietética encontrada em forragens e em outros tipos de volumosos na produção de suínos depende de vários aspectos para se tornar viável, como a identificação, a quantificação e a qualificação da fibra dietética, assim como uma avaliação de como será sua associação aos efeitos fisiológicos e ao desempenho do animal e a sua digestibilidade (Gomes et al., 2006).

Os suínos apresentam, nas regiões do cólon e do ceco, elementos fundamentais para a proliferação bacteriana, a fim de fermentar a fibra, como temperatura ótima, ambiente anaeróbio, $\mathrm{pH}$ adequado e nutrientes necessários para o bom desenvolvimento das bactérias (Berchielle et al., 2000). A celulose, a pectina e a hemicelulose são dissociadas a ácidos graxos de cadeia curta em decorrência da fase de fermentação no intestino grosso (Gomes et al., 1994).
Para determinação de produção de gases e simulação do ambiente microbiano e da digestão enzimática, podem ser utilizadas as técnicas in vitro de produção de gases (Theodorou et al., 1994). Essas se baseiam na estimativa do volume de gases produzidos por meio da leitura direta com seringas graduadas ou por conversão de volume com base em de dados de pressão (Maurício et al., 2003).

A técnica de produção de gases desenvolvida por Theodorou et al. (1994) caracteriza-se pela leitura manual do volume de gases produzidos por uma seringa plástica graduada. Maurício et al. (1999) adaptaram a metodologia desenvolvida por Theodorou et al. (1994), ao substituírem o uso de seringas para realização das leituras de volume de gás produzido por instrumentos que permitiam a captura direta de dados, o que levou à redução do tempo despendido durante as leituras, além de reduzir o erro potencial associado às mensurações.

Quando obtido o perfil de produção de gases, dispõem-se diversos modelos matemáticos para descrevê-los. Um dos modelos matemáticos mais utilizados é o de France et al. (1993). A descrição matemática das curvas de produção de gases permite a análise dos dados, a comparação dos substratos e a evolução de diferentes ambientes de fermentação, proporcionando informações sobre a composição do substrato estudado e das taxas de fermentação dos constituintes solúveis e estruturais dos substratos (Farias et al., 2011).

Ainda existem poucos estudos aplicados às técnicas de produção de gases in vitro e à influência de uma alimentação fibrosa para fermentação no ceco suíno. Assim, este trabalho teve como objetivo avaliar os padrões de fermentação microbiana do ceco de suínos criados ao ar livre, do ceco de suínos criados em sistema tradicional confinado e do líquido ruminal bovino, utilizando-se um modelo in vitro de produção de gases para ruminantes. 


\section{MATERIAL E MÉTODOS}

A pesquisa foi conduzida no Laboratório de Nutrição Animal (LNA), da Fazenda Água Limpa (FAL) da Universidade de Brasília (UnB), localizada no Núcleo Rural Vargem Bonita, Brasília, Distrito Federal (DF) a $15^{\circ} 47^{\prime}$ de latitude sul e $47^{\circ} 56^{\prime}$ de longitude oeste e altitude média de $1080 \mathrm{~m}$.

O presente estudo foi aprovado pelo Comitê de Ética do Uso Animal da Universidade de Brasília, tendo como número de protocolo: UnB Doc 119113/2011.

No ensaio, foram utilizados três tipos diferentes de inóculo para a fermentação e a análise de produção de gases: líquido extraído de ceco de suínos abatidos e criados em sistema convencional confinado (CSC) e líquido extraído de ceco de suínos abatidos e criados em sistema ao ar livre (CSF) e, como controle, o líquido ruminal de bovino fistulado (LRB).

Os substratos utilizados derivaram de uma dieta de suínos (dieta basal) à base de farelo de soja e grãos de milho, atendendo as exigências nutricionais dos animais estabelecidas pelo Nutrient... (1998) e composição centesimal dos alimentos segundo Rostagno et al. (2005). Para composição dos substratos, foram estabelecidos diferentes níveis de substituição da dieta basal pela torta da amêndoa da Acrocomia aculeata (AA), coproduto da produção do biodiesel. Os substratos foram distribuídos da seguinte forma: substrato $1-100 \%$ de dieta basal (BAS); substrato $2-90 \%$ de dieta basal $+10 \% \mathrm{AA}$ (M10); substrato $3-80 \%$ de dieta basal + $20 \%$ AA (M20) e o substrato 4 - feno de Tifton 85 (Cynodon spp.) (TF). As dietas BAS, M10 e M20 eram fareladas, e o feno de Tifton foi moído em moinho de faca tipo Wyllie.

O delineamento experimental utilizado foi inteiramente ao acaso, em arranjo fatorial $4 \times 3$, com os fatores representados pelos substratos BAS, M10, M20 e TF e pelos inóculos CSF, CSF e LRB. Os dados referentes ao potencial máximo de produção de gases (A), ao tempo de colonização em horas (L), à taxa de produção de gases $(\mu)$, à degradabilidade da matéria seca, obtidos em diferentes intervalos de tempo, foram submetidos à análise de variância, e as médias comparadas pelo teste de Tukey a 5\% de significância, por meio do software $\mathrm{SAS} \AA$

Foram utilizados, para coleta de ceco, 12 suínos, com idade média de 92 dias e peso médio de $34,8 \mathrm{~kg}$, criados em sistema ao ar livre, na Fazenda Água Limpa (UnB). Esses foram criados em piquetes com pastagem nativa e alimentados com dieta comercial. Os outros 12 cecos suínos foram obtidos de suínos criados em sistema convencional confinado em cama sobreposta, alimentados com dieta comercial, e com idade média de 150 dias com peso médio de $105 \mathrm{~kg}$. O líquido ruminal foi coletado de um bovino adulto, fistulado, mantido em pastagem de Brachiaria brizantha.

$\mathrm{O}$ ensaio in vitro de produção de gases foi realizado de acordo com Theodorou et al. (1994), com adaptações de Maurício et al. (1999). Cada inóculo foi homogeneizado e filtrado em duas camadas de tecido de algodão, sendo mantidos em diferentes balões de Erlenmeyer em banho-maria, a $39^{\circ} \mathrm{C}$, sob saturação de $\mathrm{CO}_{2}$.

Amostras de 0,5g dos substratos a serem avaliados foram pesadas e lacradas em sacos F57 ANKOM $^{\circledR}$ previamente identificados. Para a fermentação das amostras, utilizaram-se frascos de vidro com volume aferido de $160 \mathrm{~mL}$. Aos frascos, previamente identificados, foram adicionados os sacos contendo 0,5g dos substratos a serem testados conforme Beuvink e Spoelstra (1992), juntamente com 90mL de meio de cultura tamponante (Theodorou et al., 1994) e $10 \mathrm{~mL}$ do inóculo correspondente, sendo esses vedados com rolhas de borracha e lacres metálicos. Os frascos foram dispostos aleatoriamente em estufa, com circulação forçada de ar a $39^{\circ} \mathrm{C}$, sendo este considerado o tempo zero.

Foram incubados 60 frascos, 12 destes contendo apenas o inóculo e o meio de cultura tamponante como controle, utilizados para determinar a produção de gases proveniente dos conteúdos ruminal e cecal para posterior correção da produção líquida de gases. Nos 48 frascos restantes com quatro repetições de cada tratamento por inóculo, foram feitas medições até 96 horas pós-incubação. 
As leituras de pressão (PSI) para determinação da produção de gases foram realizadas com três, seis, nove, 12, 16, 24, 36, 48, 72 e 96 horas após a incubação, por meio de um transdutor de pressão modelo Press Data, conectado a uma válvula de três saídas, sendo uma saída ligada ao transdutor, outra a uma agulha $25 \mathrm{~mm} \times 0,7 \mathrm{~mm}$ e a terceira livre para remoção do gás após a leitura.

Os dados de pressão obtidos em PSI foram transformados em volume de gás produzido por meio da equação descrita por Guimarães Júnior et al. (2008), para as condições de temperatura e pressão atmosférica de Brasília, DF: volume $(\mathrm{mL})=4,50231 \mathrm{x}$ pressão $(\mathrm{PSI})+0,05164 \mathrm{x}$ pressão $^{2}\left(\mathrm{R}^{2}=0,996\right)$.

A cinética de produção de gases em cada tratamento foi determinada segundo o modelo descrito por France et al. (1993):

$$
Y=A\left\{1-\exp ^{[-b(t-L)-c x(\sqrt{ } t-\sqrt{ } L)]}\right\} \text {, em que: }
$$$$
Y=\text { produção cumulativa de gases }(\mathrm{mL}) \text {; }
$$

$A=$ potencial máximo de produção de gases $(\mathrm{mL})$;

$L=$ tempo de colonização ou lag time (h); $b\left(\mathrm{~h}^{-1}\right)$ e $c\left(\mathrm{~h}^{-0,5}\right)=$ taxas fracionais constantes; e $\mathrm{t}=$ tempo $(\mathrm{h})$.

A taxa fracional média $\left(\mathrm{h}^{-1}\right)$ de produção de gases $(\mu)$ foi calculada como:

$$
\mu=b+\_\frac{c}{2 \sqrt{t}_{t}} \text {, em que: }
$$

$\mu 48=$ taxa de produção de gases após $48 \mathrm{~h}\left(\mathrm{~h}^{-1}\right)$.

Os substratos BAS, M10, M20 e TF, utilizados no ensaio de produção de gases, foram submetidos à análise de matéria seca (MS), proteína bruta $(\mathrm{PB})$, extrato etéreo $(\mathrm{EE})$, matéria mineral (MM), lignina, fibra bruta (FB), fibra em detergente neutro (FDN) e fibra em detergente ácido (FDA). As amostras foram analisadas de acordo com a metodologia de AOAC (1995) para FDA, FB, EE, PB, MM, MS e lignina; para FDN a metodologia foi a de Van Soest et al. (1991). Os resultados obtidos das análises estão expostos na Tab. 1.

Tabela 1. Dados de análise bromatológica dos substratos estudados no ensaio de gases

\begin{tabular}{lcccc}
\hline \multirow{2}{*}{ Parâmetro (\% na MS) } & \multicolumn{4}{c}{ Substratos } \\
\cline { 2 - 4 } & TF & BAS & M10 & M20 \\
\hline Matéria seca (MS) & 83,44 & 87,53 & 86,66 & 90,38 \\
Proteína bruta (PB) & 6,65 & 26,72 & 21,27 & 21,33 \\
Extrato etéreo (EE) & 1,30 & 3,11 & 4,01 & 4,63 \\
Matéria mineral (MM) & 7,35 & 5,37 & 5,89 & 5,37 \\
Lignina em detergente ácido (LDA) & 11,99 & 1,24 & 4,57 & 5,85 \\
Fibra bruta (FB) & 35,84 & 5,60 & 6,40 & 9,90 \\
Fibra em detergente neutro (FDN) & 88,89 & 41,87 & 45,30 & 50,62 \\
Fibra em detergente ácido (FDA) & 50,34 & 15,87 & 18,55 & 21,84 \\
\hline
\end{tabular}

BAS $=100 \%$ de dieta basal, M10 $=90 \%$ de dieta basal $+10 \%$ de Acrocomia aculeata, M20 $=80 \%$ de dieta basal + $20 \%$ de Acrocomia aculeata e TF = feno de Tifton 85 (Cynodon spp.).

\section{RESULTADOS E DISCUSSÃO}

A análise de variância apresentou interação entre médias obtidas dos inóculos e dos substratos estudados, para os parâmetros produção de gases potencial (A) e tempo de colonização em horas (L).

Observa-se, na Tab. 2, que os inóculos LRB e CSF apresentaram o maior potencial de produção de gases (A), quando o substrato foi o BAS; neste caso, em que o substrato era rico em amido, os dois inóculos apresentaram importante crescimento microbiano, ajustando-se, de maneira semelhante, ao modelo proposto. No caso da M10, o inóculo LRB obteve o maior resultado, e o CSC apresentou o menor resultado
$(\mathrm{P}<0,05)$; o CSF não diferiu dos dois outros inóculos $(\mathrm{P}>0,05)$. Os valores de $\mathrm{A}$ para LRB foram maiores para o substrato $\mathrm{TF}$, mostrando a maior adaptação da microbiota do rúmen na fermentação do feno; entre o CSF e o CSC, não houve diferença para esta variável. Também não houve diferença para o potencial máximo de produção de gases entre os inóculos estudados quando o substrato foi o M20 ( $\mathrm{P}>0,05)$.

Nos resultados (Tab. 2), pode-se observar que, para os substratos BAS, M10 e M20, o inóculo de ceco suíno criado ao ar livre foi similar ao líquido ruminal bovino, indicando presença de populações microbianas semelhantes, o que pode ser observado pela proximidade entre as curvas de fermentação. Jensen e Jorgensen (1994) 
encontraram alterações no perfil de fermentação no ceco de suínos alimentados com dietas com maiores concentrações de fibra, o que poderia explicar essas semelhanças e indica a sensibilidade da técnica e do modelo adotado na avaliação de inóculos de ceco suíno.

Tabela 2. Análise de interação e teste de médias para potencial máximo de produção de gases (A) em $\mathrm{mL} / \mathrm{gMS}$, comparativo entre inóculos e substratos

\begin{tabular}{lccc|ccc}
\hline \multirow{2}{*}{ Tratamento } & \multicolumn{7}{c}{ A (mL/gMS) } \\
\cline { 2 - 7 } & LRB & CSF & CSC & CV & EPM & Valor de P \\
\hline BAS & $351,85 \mathrm{~A} \mathrm{a}$ & $337,350 \mathrm{~A} \mathrm{a}$ & $288,92 \mathrm{~B} \mathrm{a}$ & 6,282 & 20,4819 & 0,0046 \\
M10 & $294,85 \mathrm{~A} \mathrm{~b}$ & $278,80 \mathrm{AB} \mathrm{b}$ & $258,05 \mathrm{~B} \mathrm{~b}$ & 4,6692 & 12,9446 & 0,0096 \\
M20 & $288,42 \mathrm{~b}$ & $284,15000 \mathrm{~b}$ & $259,3000 \mathrm{~b}$ & 7,7604 & 21,5190 & 0,1741 \\
TF & $239,92 \mathrm{~A} \mathrm{c}$ & $116,82 \mathrm{~B} \mathrm{c}$ & $129,92 \mathrm{~B} \mathrm{c}$ & 8,9105 & 14,4551 & $<0,0001$ \\
\hline CV & 7,6993 & 6,5703 & 5,2956 & & & \\
EPM & 22,61790 & 16,7007 & 12,39450 & & & \\
Valor de P & 0,0002 & $<0,0001$ & $<0,0001$ & & & \\
\hline
\end{tabular}

Médias seguidas por letras maiúsculas diferentes na mesma linha ou letras minúsculas diferentes na coluna, em cada intervalo, diferem entre si pelo teste de Tukey $(\mathrm{P}<0,05) . \mathrm{LRB}=$ líquido ruminal bovino, $\mathrm{CSF}=$ ceco suíno criado ao ar livre, $\mathrm{CSC}=$ ceco suíno criado confinado, $\mathrm{BAS}=100 \%$ de dieta basal, $\mathrm{M} 10=90 \%$ de dieta basal $+10 \%$ de Acrocomia aculeata, M20 $=80 \%$ de dieta basal $+20 \%$ de Acrocomia aculeata e TF $=$ feno de Tifton 85 (Cynodon spp.). $\mathrm{CV}=$ coeficiente de variação, $\mathrm{EPM}=$ erro- padrão médio.

A Fig. 1 ilustra a curva de produção cumulativa de gases dos inóculos por substratos, onde se pode observar o tempo (h) de colonização das bactérias (L), desde a inoculação até o início da produção de gases, e a produção total de gases.

O comportamento dos inóculos estudados foi bem semelhante, e a população microbiana do ceco suíno se adaptou bem às condições de meio de cultura utilizada na metodologia adotada e os valores de volume de gases encontrados se ajustaram bem ao modelo de France et al. (1993).

Em todos os gráficos, verifica-se que o inóculo LRB apresentou maior volume de gases do que os demais, e a curva do CSF ficou sempre próxima deste. Os padrões de fermentação demonstraram similaridade entre esses dois inóculos, exceto quando o substrato foi o TF, em que a quantidade de fibra foi bem maior. Em todos os substratos estudados, a produção de gases foi diminuída com o aumento do FDN desses substratos (M10, M20 e TF).

Outra característica importante observada na curva de produção de gases do ceco suíno foi o tempo para produção do máximo volume de gases. Esse tempo foi próximo de 40 horas; após esse tempo, não houve aumento importante dessa produção. De acordo com Zanotto et al. (1998), o tempo de passagem do alimento pelo trato digestivo do suíno é, em média, 40 horas. Ao se analisar a Fig. 1, observa-se que, no tempo aproximado de 40 horas, a produção de gases de ambos os inóculos de ceco suíno apresentaram máxima produção (assimptótica) e mantiveram os mesmos valores até as 96 horas, indicando que, a partir desse tempo, não houve mais crescimento microbiano considerável e que o tempo de 40 horas pode ser importante para estudos de fermentação in vitro para a espécie suína.

O tempo de colonização (L) representa o tempo compreendido entre o início da incubação e o da produção de produtos da fermentação microbiana; assim, quanto menor o tempo de colonização, mais rápido o início da degradação do alimento e da produção de gases (Ribas et al., 2007). Ao se compararem os resultados entre inóculos (Tab. 3), o CSC apresentou o menor tempo de colonização quando a ração foi BAS, sendo o segundo menor tempo de CSF e o maior tempo de LRB $(\mathrm{P}<0,05)$, o que indica maior adaptação da microbiota presente no ceco de suínos confinados às dietas comerciais, mais ricas em amido.

A velocidade da colonização depende da composição da partícula, do tamanho (superfície de contato) e da afinidade do microrganismo pelo alimento (Valadares Filho e Pina 2011). De acordo com Guimarães Jr. et al. (2008), as reduções no tempo de colonização são favorecidas pela presença de substratos prontamente fermentáveis e por características físicas e químicas da parede celular da amostra. 


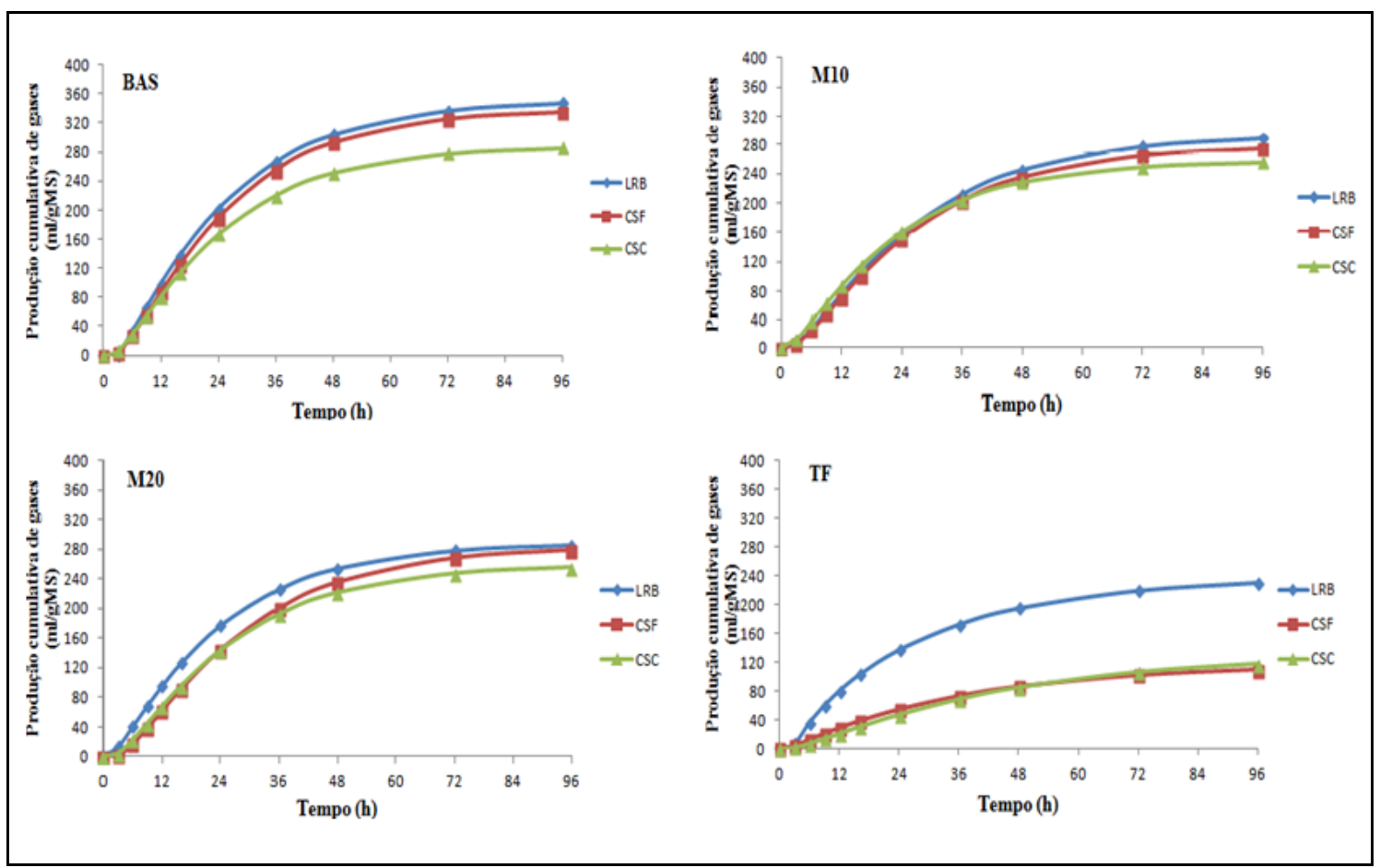

Figura 1. Produção cumulativa de gases (mL/gMS) durante 96 horas de processo fermentativo dos substratos ajustada ao modelo de France et al. (1993). BAS $=100 \%$ de dieta basal, M10 = 90\% de dieta basal $+10 \%$ de Acrocomia aculeata, M20 $=80 \%$ de dieta basal $+20 \%$ de Acrocomia aculeata e TF $=$ feno de Tifton 85 (Cynodon spp.) entre os inóculos, LRB = líquido ruminal bovino, CSF = ceco suíno criado ao ar livre, $\mathrm{CSC}=$ ceco suíno criado confinado.

Tabela 3. Tempo de colonização em horas (L), comparativo entre inóculo de rúmen bovino (RLB), inóculo de ceco suíno criado ao ar livre (CSF), inóculo de ceco suíno criado confinado (CSC) com diferentes substratos

\begin{tabular}{|c|c|c|c|c|c|c|}
\hline \multirow{2}{*}{ Tratamento } & \multicolumn{6}{|c|}{ L (h) } \\
\hline & LRB & $\mathrm{CSF}$ & $\mathrm{CSC}$ & $\mathrm{CV}$ & EPM & Valor de $\mathrm{P}$ \\
\hline BAS & $2,983 \mathrm{~A} \mathrm{a}$ & $1,764 \mathrm{~B}$ & $1,36600 \mathrm{C}$ & 9,3428 & 0,1903 & $<0,0001$ \\
\hline M10 & $0,537 \mathrm{~B} \mathrm{~b}$ & $1,345 \mathrm{~A}$ & $0,655 \mathrm{AB}$ & 36,06290 & 0,3274 & 0,0300 \\
\hline M20 & $0,883 \mathrm{~B} \mathrm{ab}$ & $2,001 \mathrm{~A}$ & $1,599 \mathrm{AB}$ & 35,03050 & 0,5235 & 0,0405 \\
\hline TF & $2,44900 \mathrm{ab}$ & 1,51400 & 1,370000 & 64,55970 & 1,1481 & 0,3918 \\
\hline $\mathrm{CV}$ & 52,62190 & 30,71140 & 36,57960 & & & \\
\hline EPM & 0,9900 & 0,5086 & 0,4565 & & & \\
\hline Valor de $\mathrm{P}$ & 0,0273 & 0,3275 & 0,0614 & & & \\
\hline
\end{tabular}

Médias seguidas por letras maiúsculas diferentes na mesma linha ou letras minúsculas diferentes na coluna, em cada intervalo, diferem entre si pelo teste de Tukey $(\mathrm{P}<0,05) . \mathrm{LRB}=$ líquido ruminal bovino, $\mathrm{CSF}=$ ceco suíno criado ao ar livre, $\mathrm{CSC}=$ ceco suíno criado confinado, $\mathrm{BAS}=100 \%$ de dieta, M10 $=90 \%$ de dieta basal $+10 \%$ de Acrocomia aculeata, M20 $=80 \%$ de dieta basal $+20 \%$ de Acrocomia aculeata e TF $=$ feno de Tifton 85 (Cynodon spp.). CV $=$ coeficiente de variação, EPM = erro-padrão médio.

Com os substratos M10 e M20, o inóculo LRB apresentou o menor tempo, CSF o maior tempo e CSC não diferiu de ambos $(\mathrm{P}<0,05)$. Não houve diferença significativa entre os inóculos para o substrato TF, indicando similaridade na capacidade de iniciar o processo de fermentação com o alimento mais fibroso entre os inóculos estudados. 


\section{CONCLUSÕES}

A técnica de produção de gases in vitro em sistema semiautomático apresentou resultados semelhantes aos encontrados para ruminantes. $\mathrm{O}$ modelo matemático utilizado foi adequado para descrever a curva de fermentação no ceco de suínos, mostrando semelhanças entre as microbiotas do ceco e do rúmen.

\section{REFERÊNCIAS}

ASSOCIATION OF OFFICIAL ANALYTICAL CHEMISTS - AOAC. Official methods of analysis. 16 ed. Washington, D.C., p.2000, 1995.

BERCHIELLI, T.T.; ANDRADE, P.; FURLAN, C.L. Avaliação de indicadores internos em ensaios de digestibilidade. Rev. Bras. Zootec., v.29, p.830-833, 2000.

BEUVINK, J.M.W.; SPOELSTRA, S.F. Interactions between substrate, fermentation end-products, buffering systems and gas production upon fermentation of different carbohydrates by mixed rumen microorganisms in vitro. Appl. Microbiol. Biotechnol., v.37, p.505-509, 1992.

BROUNS, F.; EDWARDS, S.A.; ENGLISH, P.R. Effect of dietary fibre and feeding system on activity and behaviour of housed gilts. Appl. Anim. Behav. Sci., v.39, p.215-223, 1994.

FARIAS, L.N.; VASCONCELOS, V.R.; CARVALHO, F.F.R.; SARMENTO, J.L.R. Avaliação dos modelos logístico bicompartimental e de Gompertz na estimativa da dinâmica de fermentação ruminal in vitro do farelo e da torta de babaçu (Orbigya martiana). Arq. Bras. Med. Vet. Zootec., v.63, p.136-142, 2011.

FRANCE, J.; DHANOA, M.S.; THEODOROU, M.K. et al. A model to interpret gas accumulation profiles associated with in vitro degradation of ruminant feeds. J. Theor. Biol., v.163, p.99-111, 1993.

GOMES, B.V.; QUEIROZ, A.C.; FONTES, C.A.A. Estudo das características físico-químicas de fenos de palhas. II. Efeito sobre a degradabilidade "in situ" da matéria seca, proteína bruta e fibra detergente neutro. Rev. Soc. Bras. Zootec., v.23, p.292-304, 1994.

GOMES, J.D.F.; FUKUSHIMA, R.S.; PUTRINO, S.M. et al. Efeitos do incremento da fibra em detergente neutro na dieta de suínos sobre a morfologia dos órgãos digestivos e não digestivos. Braz. J. Vet. Res. Anim. Sci., v.43, p.202-209, 2006.

GOMES, J.D.F.; PUTRINO, S.M.; GROSSKLAUS, C. et al. Efeitos do incremento de fibra dietética sobre a digestibilidade, desempenho e características de carcaça: I. suínos em crescimento e terminação. Semin.: Ciênc. Agrár., v.28, p.483-492, 2007.
GUIMARÃES JÚNIOR, R.; CABRAL FILHO, S.L.S; FERNANDES, F.D. et al. Relação entre pressão e volume para implantação da técnica in vitro semiautomática de produção de gases na Embrapa Cerrados. Fortaleza: Embrapa Cerrados, 2008. 8p. (Comunicado Técnico, n.144).

JENSEN B.B.; JORGENSEN H. Effect of dietary fiber on microbial activity and microbial gas production in various regions of the gastrointestinal tract of pigs. Appl. Environ. Microbiol., v.60, p $1897-$ 1904, 1994.

MAURICIO, R.M.; MOULD, F.L.; DHANOA, M.S. et al. A semi-automated in vitro gas production technique for ruminant feedstuff evaluation. Anim. Feed Sci. Technol, v.79, p.321-330, 1999.

MAURÍCIO, R.M.; PEREIRA, L.G.R.; GONÇALVES, L.C. et al. Potencial da técnica in vitro semi-automática de produção de gases para avaliação de silagens de sorgo (Sorghum bicolor (L.) Moench). Rev. Bras. Zootec., v.32, p.1013-1020, 2003.

NUTRIENT requirements of swine. 10.ed. Washington: National Academy, 1998. p.189.

RAMONET, Y.; MEUNIER-SALAÜN, M.C.; DOURMAD, J.Y. High-fiber diets in pregnant sows: digestive utilization and effects on the behavior of the animals. J. Anim. Sci., v.77, p.591-599, 1999.

RIBAS, R.G.; MEUNIER-SALUN, M.C.; DOURMAD, J.Y. Hihg-fiber diets in pregnant sows: digestive utilization and effects on the behavior of the animals. J. Anim. Sci., v.274, p.2399-2408, 2007.

ROSTAGNO, H.S.; ALBINO, L.F.T.; DONZELE, J.L. et al. Composição de alimentos e exigências nutricionais de aves e suínos (tabelas brasileiras). Viçosa: UFV, Imprensa Universitária, 2005. 186p.

THEODOROU, M.K.; WILLIAMS, B.A.; DHANOA, M.S. et al. A simple gas production method using a pressure transducer to determine the fermentation kinetics of ruminants feeds. Anim. Technol. Feed Sci., v.48, p.185-197, 1994

VALADARES FILHO, S.C.; PINA, D.S. Fermentação ruminal. In: BERCHIELLI, T.T.; PIRES, A.V.; OLIVEIRA, S.G. Nutrição de ruminantes. 2.ed. Jaboticabal: FUNEP, 2011. p.161-234.

VAN SOEST, P.J.; ROBERTSON, J.B.; LEWIS, B.A. Methods for dietary fiber, neutral detergente fiber and nonstarch polysaccharides in relation to animal nutrition. J. Dairy Sci., v.74, p.3583-3597, 1991.

ZANOTTO, D.L.; GUIDONI, A.L.; LIMA, G.J.M.M. Efeitos da granulometria do milho sobre a digestibilidade das dietas para suinos em crescimento e terminação. Concórdia: EMBRAPA Suínos e Aves, 1998. 2p. (Comunicado Técnico, n.223). 